\title{
Diseño de la estrategia de gestión del destino turístico regional Villa Clara (Cuba)
}

\author{
Design of the regional tourist destination management strategy of Villa Clara \\ (Cuba)

\section{Desenho da estratégia de gestão do destino turístico regional Villa Clara (Cuba)}

\author{
Giselle Rodríguez Jiménez \\ Facultad de Ciencias Económicas, Universidad Central “Marta Abreu” de Las Villas, Cuba \\ grjimenez@uclv.cu
}

\author{
Alicia de la Concepción Alfonso Serafín \\ Facultad de Ciencias Económicas, Universidad Central "Marta Abreu” de Las Villas, Cuba \\ aliciaas@uclv.cu
}

\section{Carlos Cristóbal Martínez Martínez}

Facultad de Ciencias Económicas, Universidad Central “Marta Abreu” de Las Villas, Cuba cristobalc@uclv.edu.cu

\begin{abstract}
Resumen
El objetivo de la presente investigación consiste en elaborar el procedimiento para el diseño de la estrategia de gestión de destinos turísticos regionales. Para darle cumplimiento al mismo se sentaron las bases teórico-conceptuales de la investigación en relación a los destinos turísticos y su gestión, así como la relación de estos con el diseño de estrategias, de manera que se logra proponer el procedimiento para el diseño de la estrategia de gestión de destinos turísticos regionales, estructurado en fases y etapas. Como resultados principales se presenta un procedimiento integrador y pertinente; la validación conceptual se logra mediante el juicio de expertos y la práctica mediante su aplicación en el destino turístico regional Villa Clara, Cuba. Se logra una estrategia que facilita la integración de los actores que intervienen en el destino, la eficiencia en el aprovechamiento de los recursos, así como la satisfacción en la experiencia del turista.
\end{abstract}

Palabras claves: destino turístico; estrategia; procedimiento; diseño; Villa Clara (Cuba).

\begin{abstract}
The objective of this research consists of the elaboration of the procedure for the design of a regional tourist destination management strategy. To this end, the theoretical-conceptual bases of the research in relation to tourist destinations and their management are laid out, as well as their relationship with the design of strategies, so that it is possible to propose the procedure for the design of the regional tourist destination management strategy, structured in phases and stages. The main result is a holistic and relevant procedure; conceptual validation is achieved via
\end{abstract}

Dos Algarves: A Multidisciplinary e-Journal, 36-2020.

ISBN 2182-5580 @ ESGHT - University of the Algarve, Portugal.

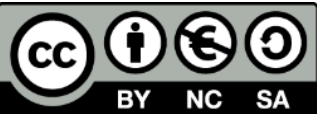

To cite this article: Rodríguez, G., Alfonso, A. \& Martínez, C. (2020). Diseño de la estrategia de gestión del destino turístico regional Villa Clara (Cuba). Dos Algarves: A Multidisciplinary e-Journal, 36, 75-96. DOI: 10.18089/DAMeJ.2020.36.5 
expert judgment and practice through its application in the regional tourist destination of Villa Clara, Cuba. A strategy that facilitates the integration of the actors involved in the destination, efficiency in the use of resources, as well as satisfaction in the tourist experience, is achieved.

Keywords: tourist destination; strategy; process; design; Villa Clara (Cuba).

\title{
Resumo
}

\begin{abstract}
O objetivo deste estudo é desenvolver o processo de desenho da estratégia de gestão de destinos turísticos regionais. Para cumpri-lo, estabeleceram-se as bases teórico-conceptuais da investigação sobre os destinos turísticos e a sua gestão, bem como a sua relação com a definição de estratégias, para ser possível sugerir o processo de definição da estratégia de gestão de destinos turísticos regionais, estruturado em diferentes fases e etapas. Os principais resultados do estudo são a apresentação de um processo holístico e relevante e a validação conceptual, por um painel de peritos, da aplicação do desenho da estratégia de gestão de destinos turísticos regionais no destino turístico de Villa Clara, Cuba. O estudo consegue propor uma estratégia que facilita a integração dos diversos atores envolvidos no destino, a eficiência no uso dos recursos e a satisfação da experiência turística.
\end{abstract}

Palavras-chave: destino turístico, estratégia, processo, desenho; Villa Clara (Cuba).

\section{Introducción}

El carácter integrador de la experiencia turística y de la satisfacción global que expresan los turistas del viaje, ha ido reafirmando el enfoque de priorizar la gestión desde los destinos turísticos. Este enfoque exige no reducir el destino a la suma de sus componentes y reconoce la complejidad que emerge del sin número de diversas relaciones de los agentes que operan en él. La gestión de destinos abarca dirigir, coordinar y apoyar la integración de diferentes recursos, actividades y agentes involucrados, a través de políticas y medidas adecuadas. Su objetivo es gestionar los diversos componentes del sistema de modo tal que asegure el cumplimiento de las metas socio-económicas que el país espera de la actividad turística con un enfoque de desarrollo sostenible.

El destino turístico constituye el principal enclave del desarrollo turístico de un territorio. En el contexto cubano su gestión ha estado limitada a las funciones de coordinación, supervisión y orientación que realizan las delegaciones del Ministerio del Turismo (Mintur) en los territorios, las que para desarrollar su trabajo cuentan con instrumentos generales de gestión turística, tales como, los planes de desarrollo, comerciales y de inversiones, entre otros.

La actual gestión del destino se caracteriza por un estilo reactivo más que predictivo o proactivo. A nivel de destino no existe un sistema formalizado e integral de información que monitoree sistemáticamente los procesos e indicadores de gestión; esta deficiencia afecta la toma de decisiones y limita prever el futuro desarrollo de los acontecimientos, estar al tanto del comportamiento de las variables y de sus valores críticos y actuar en la corrección y ajuste de políticas y estrategias. De esta problemática se deriva la interrogante: ¿cómo contribuir a la gestión estratégica del destino turístico regional Villa Clara? 
Para dar respuesta a la anterior situación es que se propone como objetivo en la presente investigación diseñar la estrategia de gestión del destino turístico regional Villa Clara; debido a la necesidad, importancia y actualidad que la determinación, y sobre todo la utilización, de una adecuada estrategia de desarrollo tiene para la gestión eficiente de un destino turístico. Se logra una adecuada gestión del destino turístico regional con un carácter de integración de los actores que intervienen, aprovechando eficientemente sus recursos y atrayendo al mayor número de turistas al mismo de modo que satisfagan sus necesidades.

\section{Revisión de la literatura}

El análisis del turismo, implica la utilización temporal de un espacio distinto al de la residencia habitual, donde se pretende desarrollar un conjunto de actividades recreativas a partir del uso de cualquier elemento natural, actividad humana o producto antropológico que pueda motivar el desplazamiento hacia dicho espacio con el móvil esencial de la curiosidad o la posibilidad de realizar una actividad física o intelectual (Rodríguez, 2005), lo cual constituye el recurso turístico.

Tanto el espacio natural como el medio social y cultural constituyen recursos turísticos que pueden convertirse en atractivos o facilidades para el desarrollo de la actividad (Salinas, 2003).

El escenario turístico incluye la combinación de los recursos naturales y antrópicos en un todo único. Esto es lo que constituye el elemento fundamental del turismo: los atractivos turísticos. Por tanto, el turismo siempre está vinculado a un cierto espacio objetivo y subjetivo a la vez, "mezcla de lo natural y lo antrópico e incluso de lo virtual", denominado en su conjunto espacio turístico (Salinas, 2003: 292).

En la actualidad, existen diversos puntos de vista en la conceptualización del término espacio turístico. Así, partiendo de la acepción más simple y general: "lugar geográfico determinado donde se asienta la oferta turística y hacia donde fluye la demanda" (OMT, 1998: 54), se podría recurrir a múltiples definiciones abordadas por diferentes estudiosos del tema, los que coinciden en que se trata de un espacio geográfico concreto y objetivo, de dimensiones y formas variables, donde concurren recursos y atractivos favorables para el desarrollo de la actividad turística y donde se establece una infraestructura y un conjunto de instalaciones, propiciadoras de esa actividad (Rodríguez, 2019).

Dado que los destinos se consideran un conjunto de productos individuales que integran atractivos naturales y artificiales, lo cual constituye la principal razón de viaje del turista (Machado, 2011); puede desarrollarse un destino en cada uno de los tipos de espacios turísticos y a su vez puede abarcar más de un tipo de espacio o la totalidad.

El estudio de las principales aportaciones de varios autores, independientemente de los distintos enfoques abordados, posibilitó identificar los elementos más representativos en el concepto de destino turístico. El análisis permitió agrupar a los autores en función de las variables que integran, constituyendo el concepto propuesto por Valls (2004) el más adecuado a los objetivos de la investigación. Este autor define destino turístico como un: Espacio geográfico determinado, con rasgos propios de climas, raíces, infraestructura y servicios, y con cierta capacidad administrativa para desarrollar instrumentos comunes de 
planificación, que adquiere centralidad atrayendo a turistas mediante productos perfectamente estructurados y adaptados a la satisfacción buscada, gracias a la puesta en valor y ordenación de los atractivos disponibles; dotados de una marca, y que se comercializa teniendo en cuenta su carácter integral (Valls, 2004).

El destino debe ser entendido como una realidad sistémica compleja, con una dialéctica propia donde se integran al mismo tiempo los elementos que conforman el sistema turístico y los elementos del sistema territorial, así como, las relaciones que entre ellos se establecen (Rodríguez, 2011).

Por otra parte, conviene utilizar la clasificación de Martín (2010) dado que incluye las diferencias que pueden aparecer según los niveles geográficos y del propio desarrollo evolutivo del turismo. Esta clasificación es aceptable debido a que permite identificar cada espacio turístico según el nivel de desarrollo que haya alcanzado. Los niveles de desarrollo, siempre acorde a la complejidad de su composición, se pueden clasificar como (Table 1):

Table 1. Clasificación del producto territorial según el nivel de desarrollo

\begin{tabular}{|l|l|c|}
\hline \multicolumn{2}{|c|}{ Nivel de Desarrollo del Produto Turístico Territorial } \\
\hline 1 & Bajo & Complejo Turístico \\
\hline 2 & Medio - bajo & Polo Turístico o Zona \\
\hline 3 & Medio & Destino Específico \\
\hline 4 & Medio - Alto & Región Turística \\
\hline 5 & Alto & Destino País \\
\hline
\end{tabular}

Fuente: Martín (2010).

Los territorios donde actúa de modo preferente o exclusivo el turismo no son una empresa. En los destinos turísticos están presentes diversas estructuras empresariales e institucionales que hacen de los mismos un sujeto complejo, dada la interacción de tales formas organizativas. Es en ese contexto donde adquiere importancia la gestión. Partiendo de la acepción más simple y general que define la gestión como "la dirección de las acciones que contribuyen a tomar decisiones orientadas a alcanzar los objetivos trazados, medir los resultados obtenidos, para finalmente, orientar la acción hacia la mejora constante del sistema" (Campaña, 2003, citado por Navarro, 2012: 20).

El Colectivo de Autores de la Universidad de Habana (2011) plantean la necesidad de una cierta estructura administrativa, un "sujeto o ente gestor" territorial, que resuelva los problemas de carácter horizontal que plantea el fenómeno turístico, que, con el apoyo de instancias de gobierno, pueda lograr la integración y coordinación de los distintos agentes que intervienen en el desenvolvimiento del turismo.

López y López (2007) coinciden con estos argumentos y confirman el carácter integrador de la gestión al afirmar que una tarea fundamental de los responsables del destino turístico se ocupa precisamente de "integrar los distintos recursos, bienes y servicios, equipamientos e infraestructuras que afectan directa o indirectamente a la actividad turística, de manera que se diseñe una planificación común y se realice una gestión conjunta de todo este sistema turístico". 
Por ende, se puede afirmar que la Gestión Integrada en Destinos Turísticos son todas aquellas actividades coordinadas para planificar, organizar, dirigir y controlar como un todo un destino turístico, con el fin de articular los componentes de su oferta y establecer una plataforma de gestión eficiente de manera que permita configurar un producto turístico identificable, competitivo y diversificado, y generar valor añadido con la correcta promoción y gestión del destino turístico. De forma general, es la acción y efecto de gestionar, bajo preceptos de integración, la actividad turística en un destino.

El proceso de gestión integrada de destinos turísticos se apoya en la planificación estratégica del destino como base para la toma de decisiones y proyección de estrategias que se centren en la satisfacción de los implicados (tanto los actores como los turistas) con base en los principios de la integración y del desarrollo sostenible, que contribuya con la competitividad del destino, respondiendo a las aspiraciones sociales y protegiendo el entorno cultural y natural.

El diseño de la estrategia implica la articulación dialéctica entre los objetivos (metas perseguidas) y la metodología (vías instrumentadas para alcanzarlas). En este sentido, la literatura recoge un gran número de procedimiento planteados para llevar a cabo procesos estratégicos.

Luego de analizados y comparados estos procedimientos (Rodríguez, 2019) se puede afirmar que el $100 \%$ de los autores consideran decisivo el diagnóstico en el destino, mientras que el 38.5\% de los mismos especifican indicadores de diagnóstico. Además, es importante resaltar cómo algunos de ellos incluyen dentro de su diseño el Análisis DAFO (debilidades, amenazas, fortalezas y oportunidades). El 69.2\% comparten el criterio de la determinación de objetivos como pauta para la definición de estrategias, cuestión en la que todos están de acuerdo como parte del procedimiento. El 84.6\% de ellos coinciden en la programación de acciones y en un posterior seguimiento y control del cumplimiento (76.9\%). Todo lo anterior sobre la base de un proceso previo de organización del trabajo (61.5\%).

Después de analizados los procedimientos para el diseño de estrategias, se plantea la necesidad del procedimiento para el diseño de estrategias de gestión de destinos turísticos regionales. Este va a considerar las etapas de mayor impacto en el análisis anterior e incorporará otras de suma importancia para la particularidad de los destinos turísticos regionales, de esta manera queda diseñado un procedimiento de gran actualidad y flexibilidad.

La puesta en práctica de modelos de desarrollo de carácter integrado, no solo permitirá a los diferentes sectores y subsectores económicos avanzar en un mismo sentido, facilitar el mantenimiento y la conservación del medio físico-natural y cultural, y de esta forma lograr el ordenamiento territorial de los espacios con potencial turístico, sino también la implantación de metodologías de planificación turística basadas en estrategias globales integradas que potencien el desarrollo en general. De esta forma se logrará que la actividad turística participe un sistema integrado, bajo una perspectiva más amplia que no se limita solamente a la relación oferta-demanda; sino que se constituye como un sistema socio-económico, dinámico y funcional. 


\section{Metodología}

Los métodos que propician el desarrollo de la investigación se analizan desde enfoques teóricos con el empleo de la inducción-deducción, el análisis-síntesis y el histórico-lógico. También empleados son las técnicas de recolección de datos como es el caso de las entrevistas estructuradas y no estructuradas y la observación, las cuales aportan el inmenso cúmulo de información necesaria para el desarrollo de la investigación. Se realizó un fuerte trabajo estadístico con la ayuda del programa informático IBM SPSS Statistics (Statistical Package for the Social Sciences) para el procesamiento de toda la información y se utilizaron métodos matemáticos para el cálculo de los índices utilizados en la investigación.

El procedimiento que se propone aplicar en el destino turístico regional involucra como primera etapa la organización de un equipo de trabajo, de manera que se resalta el carácter participativo del mismo, estos deberán trabajar en conjunto en el transcurso de todas las fases del procedimiento.

Se apoya además en un diagnóstico estratégico, fase de suma importancia por constituir una de las que más impacto obtuvo durante el análisis de los procedimientos para diseñar estrategias, este diagnóstico incluye dentro de sus pasos análisis de elementos tanto externos como internos del destino, el carácter integrador y sistémico de la gestión, así como, modelos de desarrollo, que permite establecer no solo el estado actual sino la proyección futura que pueda tener el destino, concluyendo exitosamente con un análisis estratégico, base del posterior diseño de la estrategia, que tendrá lugar en la tercera fase del procedimiento.

Importante resulta establecer la misión y la visión del destino, aporte máximo a la sociedad, el cual resulta una guía para el establecimiento de objetivos estratégicos y del posterior plan estratégico. Otro elemento a destacar en el procedimiento es la sensibilización de los implicados en la estrategia, este proceso además de resaltar el carácter participativo lo hace flexible y dinámico, pues esta constituye la etapa previa a la ejecución de las acciones estratégicas. Por último la evaluación y mejora permite la retroalimentación necesaria de lo planificado con la realidad, pudiendo ser modificadas etapas para el logro de un desarrollo armónico, al mismo tiempo que permite controlar las variables estratégicas del destino y constituir la base para la toma de decisiones o posteriores acciones estratégicas.

El procedimiento consta de 5 fases y 17 etapas (Figura 1 ).

Para la aplicación del procedimiento es necesario cumplir ciertas premisas:

1. Estar en presencia de un destino turístico regional;

2. Interés de la alta dirección;

3. Objetivos del estudio bien definidos;

4. Periodicidad del estudio determinada; $y$

5. Disponibilidad de recursos para el estudio. 
Figura 1. Procedimiento para el diseño de la estrategia de gestión de un destino turístico regional

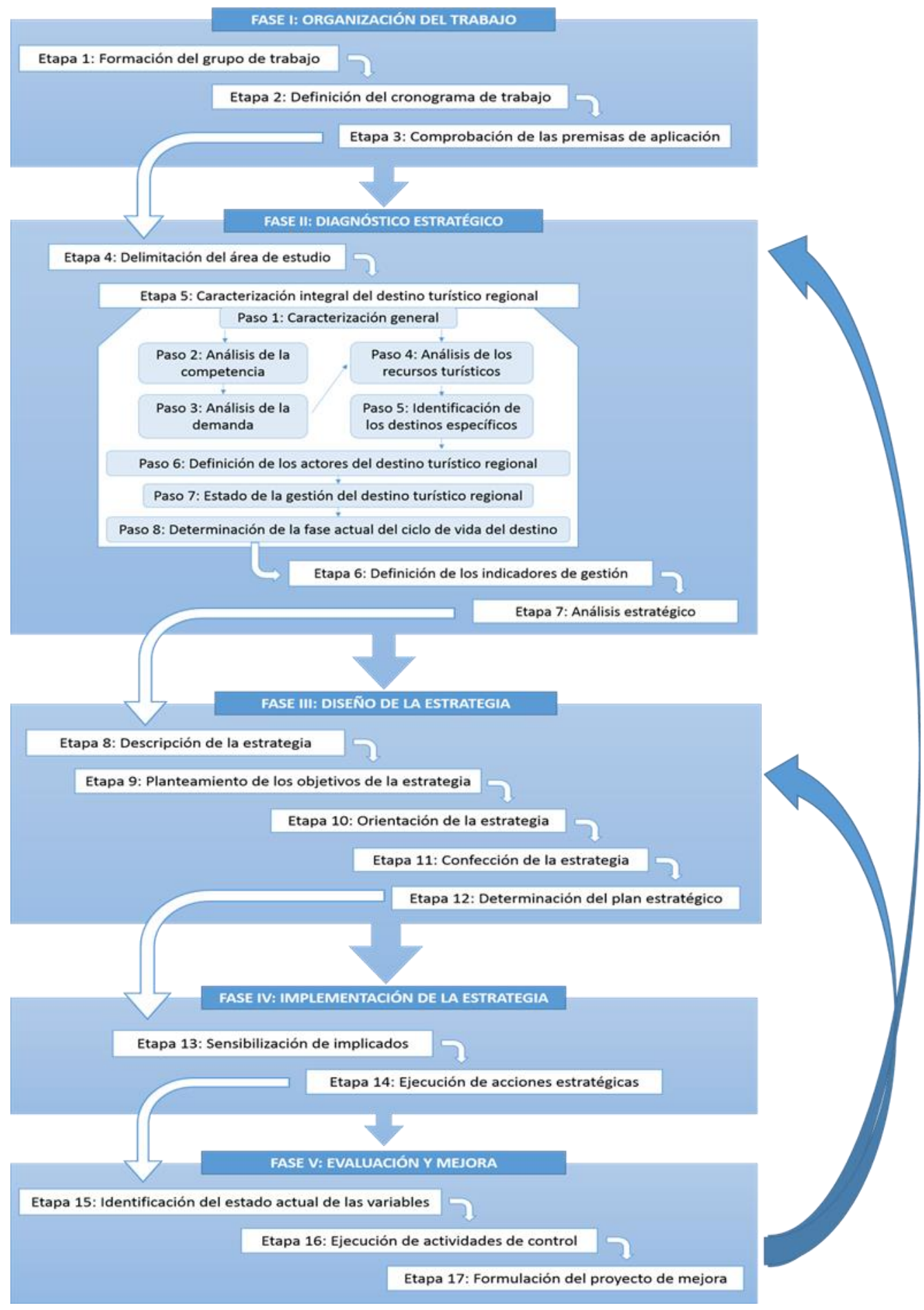

Fuente: Rodríguez, 2019.

El procedimiento desarrollado se sustenta en los siguientes principios: Lógico, porque es coherente en sus fases y permite su aplicación destinos turísticos regionales; Prospectivo, 
a pesar de presentarse en escenarios con incertidumbre y no linealidad permite establecer una estrategia para destinos turísticos regionales a largo plazo; Pertinente, el procedimiento desde su origen permite concluir de manera útil con una estrategia para destinos turísticos regionales; Incluyente, por permitir la participación de entes interesados desde su diseño hasta su implementación; Integrador, posee variables que se vinculan de manera multilateral para abordar objetos de estudio de la investigación; Transparente y con parsimonia, por permitir su entendimiento claro, sucinto y concreto; y Versátil, porque obedece a las condiciones del destino turístico regional.

El procedimiento tiene por objetivo lograr una ventaja competitiva del destino sobre la base del diseño de una estrategia de gestión a través de la participación de los actores, logrando la explotación de los recursos turísticos históricos, culturales y naturales de forma integrada sin perder la esencia de la visita del cliente, donde se pongan en marcha acciones a nivel de las estrategias funcionales (investigación y desarrollo; comercialización; finanzas; logística y servucción).

\section{Análisis y discusión de los resultados}

Se decide orientar la validación del procedimiento mediante el Juicio de expertos y su aplicación práctica al destino turístico regional Villa Clara, Cuba. De esta forma se demuestra la viabilidad y validez del procedimiento, a partir de la consecución de las diferentes etapas que se plantean y el empleo de las herramientas de análisis necesarias para su desarrollo exitoso.

Para aplicar el juicio de expertos como parte de la validación del procedimiento propuesto se siguieron los pasos definidos a partir de la adecuación del criterio de De Arquer (2006). La opinión de los expertos confirma que el procedimiento: está contextualizado, tiene continuidad y carácter integrador, posee racionalidad, aplicabilidad, consistencia lógica, flexibilidad, perspectiva y pertinencia, crea un ambiente participativo que motiva la puesta en práctica del plan como resultado de la aplicación del procedimiento, existe disponibilidad de información requerida para su aplicación y tratamiento y cada uno de los pasos propuestos es conveniente.

La evaluación del procedimiento por los expertos, concluye que la concordancia entre los mismos no es casual, y que los criterios del grupo, acerca del procedimiento, son favorables y contribuirán a la aplicación efectiva del mismo en la estrategia de gestión de destinos turísticos regionales.

Una vez validado el procedimiento por los expertos se procede a la aplicación del procedimiento propuesto para el diseño de la estrategia de gestión de destinos turísticos regionales, adoptando como caso de estudio al destino Villa Clara, Cuba.

\section{- Fase I: Organización del trabajo}

En esta primera fase se realizaron las tres primeras etapas del procedimiento, donde primeramente queda conformado el grupo de trabajo por cinco personas: tres de ellas profesoras-investigadoras del Departamento de Turismo de la Universidad Central "Marta 
Abreu" de Las Villas que tienen como línea de investigación la gestión de los destinos turísticos, así como dos personas directivos de la delegación provincial del Ministerio del Turismo en Villa Clara. Se elaboró un cronograma de trabajo que permite la ejecución de las fases y etapas de forma ordenada, abarcando un período de tiempo de dos años y medio; y por último quedaron comprobadas las premisas para la aplicación del procedimiento, y con ello se da paso a las siguientes fases del procedimiento.

\section{- Fase II: Diagnóstico estratégico}

\section{Etapa 4: Delimitación del área de estudio}

El área de estudio comprende la provincia de Villa Clara, la cual limita con las provincias de Sancti Spíritus y Cienfuegos. Hacia el oeste lo hace con la provincia de Matanzas.

\section{Etapa 5: Caracterización integral del destino}

- Paso 1: Caracterización general

En este paso se realiza una breve caracterización de la región en cuanto a geomorfología, clima, demografía y se identifican las principales potencialidades del territorio.

- Paso 2: Análisis de la competencia

Este análisis se realiza sobre el modelo de las cinco fuerzas de Porter (1985), teniendo en cuenta tres de estas: la rivalidad entre competidores, la amenaza de competidores potenciales y la amenaza de productos sustitutivos para el destino turístico regional Villa Clara.

\section{- Rivalidad entre competidores}

Cancún, Riviera Maya, Punta Cana y Ciudad México, son los destinos que en estos momentos representan una amenaza directa para Cuba. Se reconocen como destinos competidores para Villa Clara: La Habana, Matanzas, Ciego de Ávila y Holguín. No obstante, estos representan posibles alianzas para la creación de un producto integrado.

\section{- Amenaza de Competidores potenciales (nuevos competidores entrantes)}

Entre los competidores potenciales del destino Cuba se encuentran Puerto Rico y Jamaica. Asimismo, los destinos de Guyana, Belice, Islas Caimán, Granada y Bahamas presentan crecimientos acelerados. Para el destino Villa Clara puede constituir un competidor provincial Camagüey por presentar este destino regional proyecciones en el desarrollo turístico de características multimodal.

\section{- Amenaza de productos sustitutivos}

No existe amenaza de productos sustitutivos dado que hasta el momento no se conoce alguno capaz de reemplazar la diversidad de modalidades, servicios y productos que engloba el destino turístico regional Villa Clara. A pesar de ellos se le debe prestar atención a los 
destinos regionales cubanos que satisfacen las necesidades de los turistas respecto a una modalidad específica.

\section{Paso 3: Análisis de la demanda}

Para el análisis de la demanda se aplicaron encuestas a los turistas que arribaron al destino turístico regional Villa Clara, hospedados en su mayoría en los destinos turísticos específicos de Caibarién, Remedios y Santa Clara; obteniéndose, una vez procesadas las mismas, una caracterización general de la demanda en el territorio.

La concentración por nacionalidades es muy alta con respecto a los canadienses (76\%), en mayor medida que en el conjunto de Cuba (35\% aproximadamente), otros mercados importantes son Alemania, Italia, España y Francia. Sin embargo, ninguno de ellos alcanza el $3 \%$ de pernoctaciones en el destino regional.

Respecto a la organización del viaje, un $45.7 \%$ prefiere organizar su viaje de manera individual, mientras que un $50.8 \%$ lo hace a través de las agencias de viaje.

La composición etaria mayoritaria (43\%) está en el rango de 41-60 años. Le sigue el rango de 26 a 40 años con un $28.3 \%$ y luego el de 15 a 25 años con un $19.5 \%$. El rango de la tercera edad alcanza tan solo un $8 \%$.

La distribución por género muestra que el sexo que predomina es el femenino con un $54.8 \%$ de presencia en el destino, encontrándose estas entre los grupos de edades de 15 y 25 , y, 41 y 60 años respectivamente, mientras que al sexo masculino corresponde fundamentalmente el grupo de los adultos mayores (61 años o más). En general, se reconoce que las mujeres con familia inciden en la toma de decisiones de viajes familiares.

Respecto a los motivos de viaje y los atractivos-facilidades preferidos en el destino, el $48.2 \%$ viajan por sus playas. Un $18.8 \%$ se motiva por entrar en contacto con el pueblo. Otros, de bajos ingresos en su mayoría, se alojan en hostales, y vienen interesados por la cultura y la historia, ascienden al $10.8 \%$ de los alojados. La seguridad en el destino es un aspecto que se ha convertido de suma importancia para el turista actual. Los clientes se muestran satisfechos con la visita a Villa Clara pues un $84.5 \%$ muestra intenciones de regresar y un $89 \%$ lo recomendaría a sus amistades.

La estacionalidad también constituye una variable que es necesario controlar. A partir de este análisis de la curva de Lorenz y el Índice de Gini se puede concluir que los meses de baja turística son junio, octubre, mayo y septiembre, mientras que diciembre, enero, febrero y marzo, pueden considerarse como meses de alta turística.

\section{Paso 4: Análisis de los recursos turísticos}

Al tratarse de un destino turístico regional, el cual abarca gran diversidad de recursos y atractivos, se sigue la estructura definida en bloques desarrollada por Rodríguez (2005) en su procedimiento para inventariar recursos turísticos. A continuación, se presentan los principales resultados obtenidos:

- Bloque 1: Recursos Naturales

Al considerar los elementos: fauna, vegetación, geomorfología y paisaje. En el territorio prevalece un relieve de llanuras y alturas. Se destacan varios ríos bien identificados así como 
presas importantes; a los que se suman los manantiales minero medicinales de Elguea, famosos por las propiedades curativas de sus aguas. Para inventariar los recursos turísticos naturales se tuvo en cuenta la estratificación de la provincia en tres regiones (Sagua, Caibarién y Costa Sur) como parte del ordenamiento turístico del territorio. Finalmente fueron identificados un total de 38 recursos naturales.

- Bloque 2: Recursos históricos - culturales

Se consideran los siguientes aspectos: arquitectura popular, arquitectura monumental, artesanía, manifestaciones artísticas, folklore, fiestas y tradiciones, ferias y eventos, gastronomía y urbanismo. La arquitectura villaclareña, cuenta con una fuerte influencia española, con un predominio de estilos neoclásico y art decó. Mitos y leyendas escriben la historia Villa Clara y la fundación de sus ciudades. Fueron identificados los principales eventos que se llevan a cabo en la provincia. Existen sedes de eventos reconocidas en la provincia. En la gastronomía también destacan algunos platos típicos de la región.

- Bloque 3: Infraestructura Turística.

Se consideran los siguientes aspectos: alojamiento, restauración e instalaciones de ocio. Las cadenas hoteleras que operan en Villa Clara son Gaviota, Islazul, Cubanacán y Campismo. Actualmente la provincia cuenta con una capacidad de 12477 habitaciones. A esta oferta se suma los alojamientos del sector privado. Para la restauración se cuenta con entidades de la Sucursal Palmares, instalaciones del sector privado y otros establecimientos pertenecientes a otros ministerios. Las instalaciones de ocio más reconocidas del territorio se encuentran en los municipios de Santa Clara, Placetas, Sagua La Grande, Remedios y en Cayo Santa María. - Bloque 4: Infraestructura de equipamiento (servicios)

En Villa Clara están presentes las entidades Extrahoteleras: Tiendas Caracol; Transtur y Turarte. A estos se añade la Marina Marlín de Isabela de Sagua. Entre las agencias de viajes se encuentran: Viajes Cubanacán, Cubatur, Havanatur, Ecotur y Gaviota Tours. Las entidades de apoyo son: "Comercializadora ITH S.A.", Servisa, Emprestur Villa Clara, Inmobiliaria del Turismo y la Empresa de Servicios Informáticos Especializados. Además, el destino cuenta con gasolineras, casas de cambio, bancos, hospitales, farmacias, librerías, servicios de taxi, servicios de mensajería y teléfonos, red de transporte como ómnibus, ferrocarril y aeropuerto, así como centros de información. A esto se le suman cines, teatros, galerías, museos, bibliotecas y escuelas.

\section{Paso 5: Definición de los destinos específicos}

Los destinos específicos identificados en el destino turístico regional Villa Clara responden a Caibarién, Remedios, Santa Clara, Sagua la grande y Manicaragua, debido a que ellos poseen recursos turísticos que son atractivos para los turistas y están siendo puestos en valor para su uso y conservación; cuentan además con una infraestructura de apoyo para el desarrollo de la actividad, por tanto generan flujos de visitantes hacia ellos. Para visualizar los mismos se diseñó el modelo horizontal del desarrollo del destino Villa Clara (Figura 2). 
Figura 2. Modelo de desarrollo horizontal destino turístico regional Villa Clara

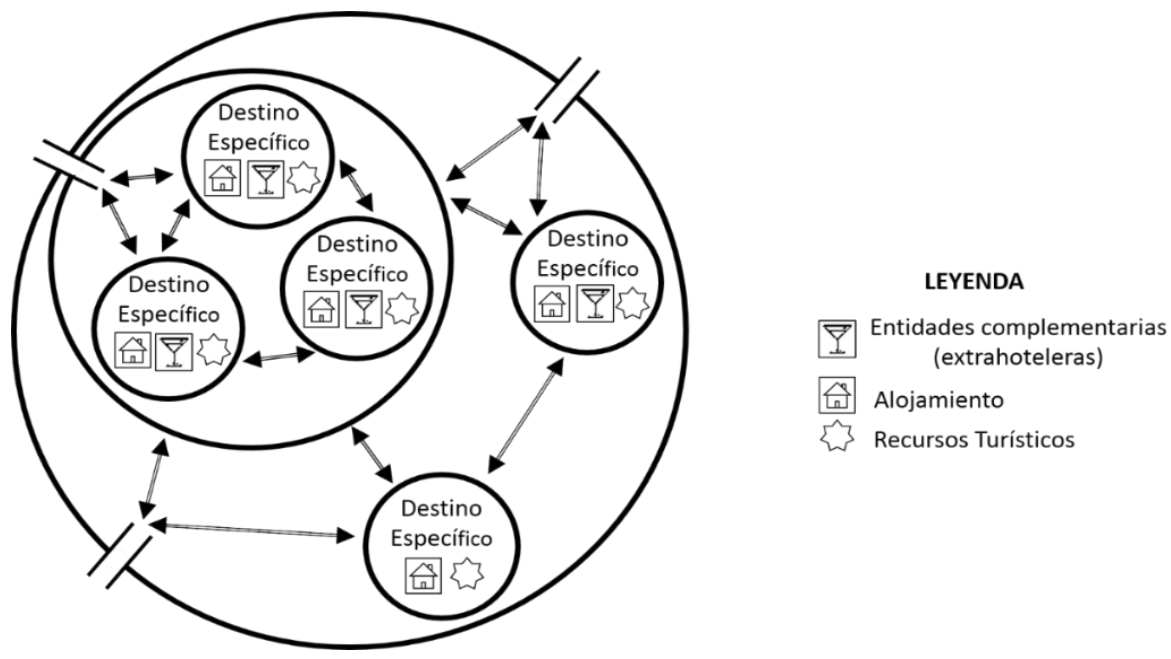

Fuente: Elaboración propia.

De esta manera se pueden identificar en el modelo varios elementos claves: Las fronteras (límites territoriales representados por el círculo mayor), las puertas de entrada (entrada de turistas al destino, se identifican vías aéreas, terrestres y marítimas; representadas por las líneas paralelas), las rutas de movilidad (flechas de doble punta, significan los flujos de turistas en el destino regional) y los servicios y recursos turísticos significados en la leyenda. Importante en el modelo de desarrollo horizontal resulta la definición de los nodos (destinos turísticos específicos, identificados con los círculos más pequeños). Las relaciones sinérgicas entre varios de estos nodos y el resto del territorio son las que generan la masa crítica suficiente como para posicionarse eficazmente en el mercado, lo que vendría siendo el destino turístico regional Villa Clara.

\section{Paso 6: Definición de los actores del destino}

En esta etapa se realizó un estudio de los actores en el destino a través de las relaciones que se establecen entre ellos en el proceso productivo del turismo. En un primer momento se identificaron los actores, teniendo en cuenta los agentes interesados identificados por Machado (2011):

- Administración Local: Consejo de la Administración Provincial de Villa Clara;

- Sector Público: Mintur Villa Clara, Universidad Central "Marta Abreu" de Las Villas (UCLV), Centro de Capacitación del Turismo (CCT), Delegación del Ministerio de Ciencia, Tecnología y Medio Ambiente (CITMA), Oficina Nacional de Administración Tributaria (ONAT), Empresa de Acueducto y Alcantarillado de Villa Clara (EAAVC), Comercio y Gastronomía, Dirección Provincial de Servicios Comunales, Delegación Provincial del Ministerio de la Agricultura (MINAG), Unión Eléctrica (UNE) y Empresa de Telecomunicaciones de Cuba S.A. (ETECSA);

- Empresas Turísticas: Islazul, Cubanacán, Gaviota, Campismo Popular, Palmares, Tiendas Caracol, Transtur, Transgaviota, Turarte, Infotur, Emprestur, Empresa de 
Servicios Informáticos Especializados (GET), Inmobiliaria del Turismo, Comercializadora ITH S.A, y Servisa;

- Intermediarios: Viajes Cubanacán, Cubatur, Havanatur, Ecotur y Gaviota Tours;

- Comunidad Local: (Habitantes de la provincia, Negocios privados de restauración y hostales);

- Turistas: (Turistas que visitan la provincia).

Luego se presentan de forma gráfica, los resultados de la investigación de las relaciones entre los 36 actores identificados (Figura 3). De esta manera, las flechas indican entre cuáles de ellos se producen interacciones, o vínculos, para la ejecución de actuaciones relacionadas con la gestión del destino. A partir del análisis de la red de actores turísticos, la primera característica significativa tiene que ver con la centralidad de la red, que se encuentra liderada por el Mintur, pues está unido directamente a un mayor número de actores y, además, impulsa las interacciones con el resto de actores de la red.

Otra característica de esta red es la gran representación de actores, en su mayoría del sector público, cuya implicación está más relacionada con su capacidad de influencia, al ser requeridos por los otros actores, que por ser impulsores de relaciones, además se detecta una cierta pasividad de algunos que casi exclusivamente presentan grado de centralidad de entrada, a la par, existe una pobre implicación de los habitantes en el accionar turístico que se ve limitado a la interacción con los turistas y a su vinculación con los negocios privados de restauración y alojamiento. Destaca además, la gran dependencia de las agencias de viaje receptivas como canales de distribución y medios de integración del producto global del destino en el país.

Figura 3. Dinámica relacional en la gestión turística de Villa Clara

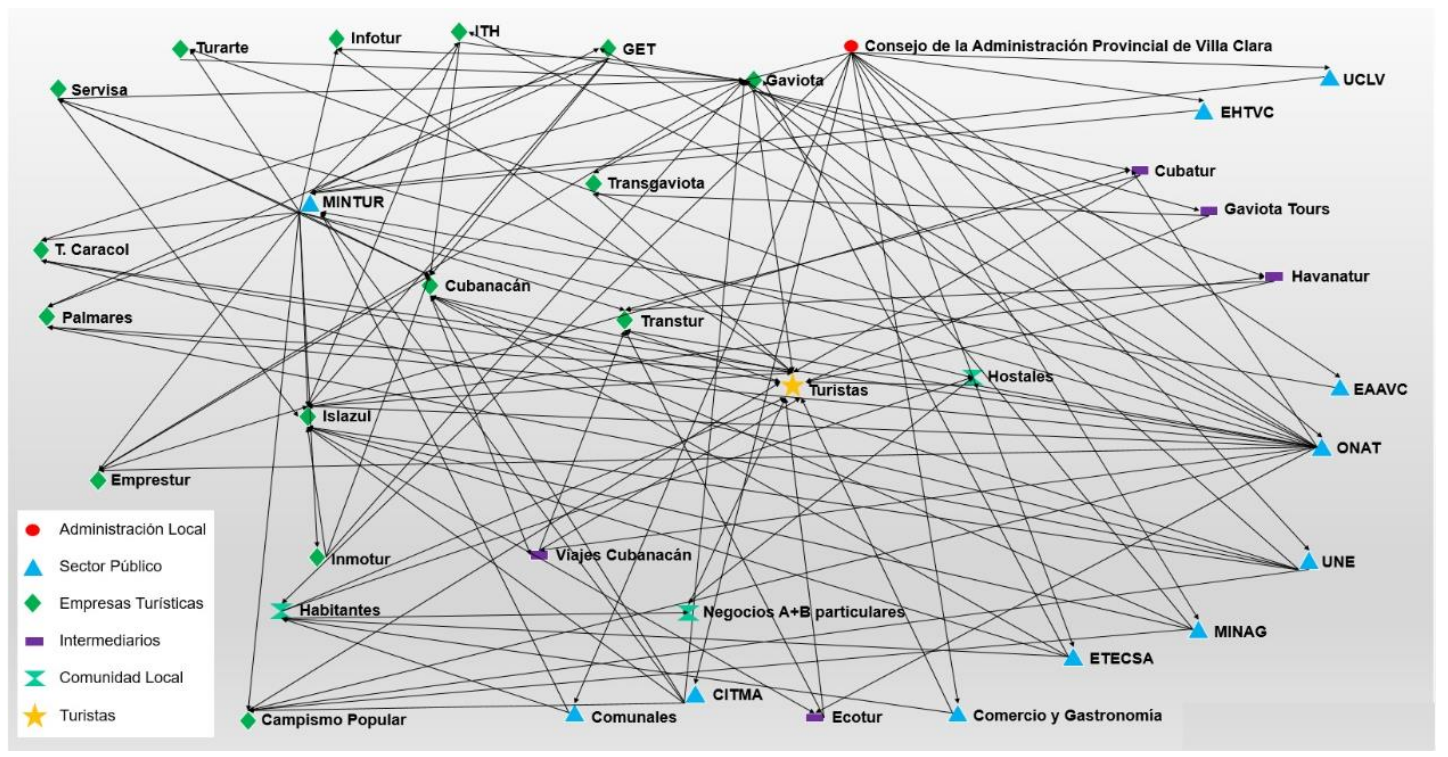

Fuente: Elaboración propia. 


\section{Paso 7: Estado de la gestión del destino turístico regional}

El posicionamiento del destino Villa Clara decayó notablemente tras el paso del huracán Irma en septiembre de 2017. Debido a esto, los esfuerzos en la comercialización del mismo se redoblaron en FitCuba2018 para promover el producto de sol y playa en Cayo Santa María. Dado los resultados obtenidos luego de la feria sigue siendo una prioridad para el territorio desarrollar acciones comerciales que incentiven la modalidad.

Otro aspecto importante en la gestión del destino lo constituye el análisis de este como sistema a través de un clúster. La dinámica relacional elaborada como parte del análisis de los actores, sentó las bases para la elaboración del Clúster Potencial del Turismo en el destino turístico regional Villa Clara (Figura 4), debido a que los participantes aparentemente carecen de la implicación necesaria para trabajar en conjunto hacia un fin común.

Figura 4. Clúster potencial del turismo en el destino turístico regional Villa Clara

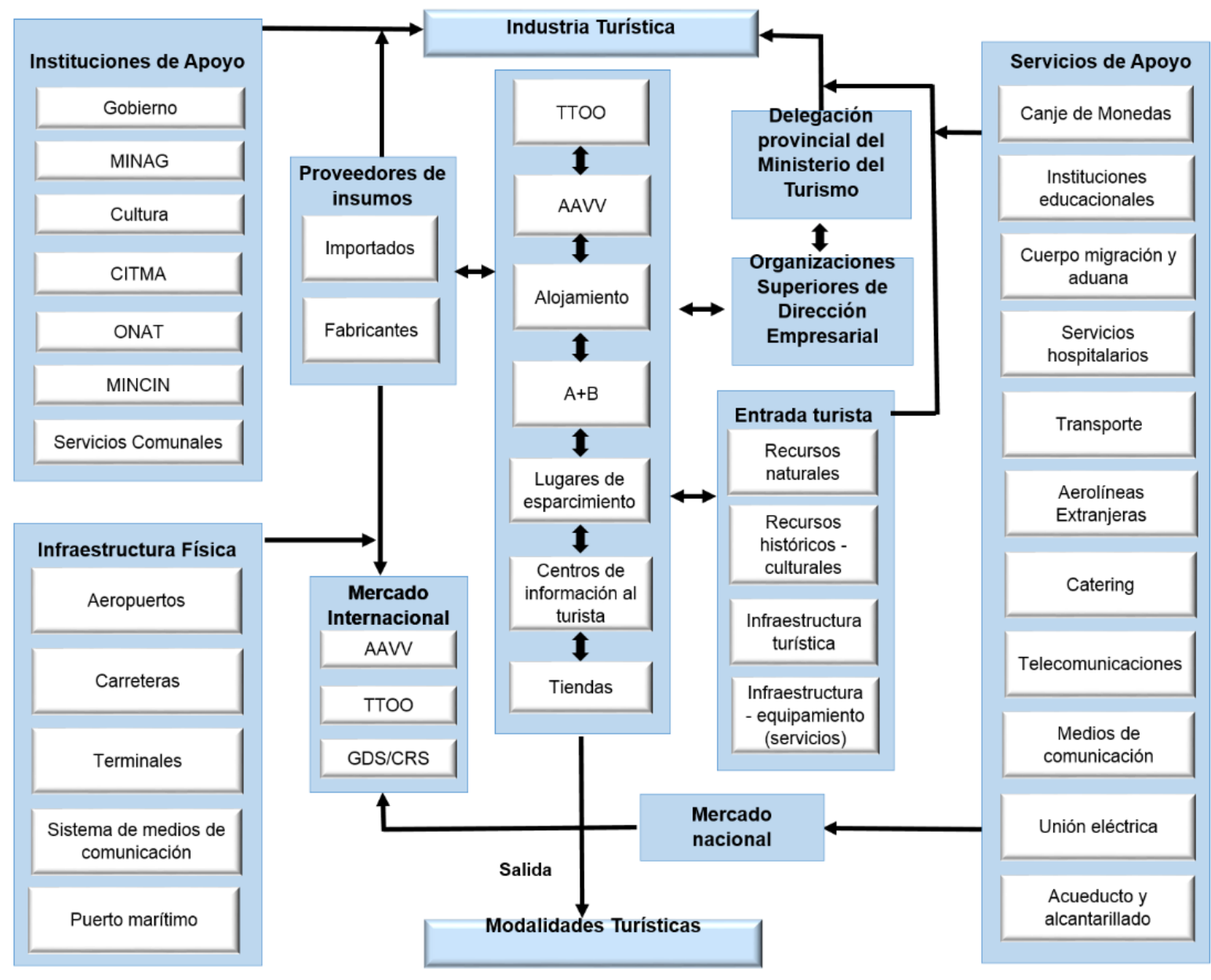

Fuente: Elaboración propia. 
En este sentido la industria turística cuenta con la dirección del Mintur, quien entabla relaciones de retroalimentación con las Organizaciones Superiores de Dirección Empresarial (Cubanacán, Islazul, Campismo, Gaviota, Cubasol, Viajes Cuba y Servitur). Estas a su vez mantienen una línea de retroalimentación con las empresas directamente relacionadas a la actividad turística, y estas últimas con los recursos naturales, histórico culturales, de infraestructura turística y de equipamiento. Estos recursos potencian el grado de atractividad del destino por lo que su influencia es directa. Asimismo, los servicios de apoyo enriquecen la entrada de turistas e influyen sobre el mercado. Este último guarda relación con la infraestructura física y los proveedores de insumo, quienes se retroalimentan con las empresas turísticas y por tanto se relacionan directamente con la industria turística, quien a su vez recibe la influencia de las instituciones de apoyo. Finalmente, todas estas relaciones conducen al desarrollo de diferentes modalidades turísticas en el territorio.

Por último resulta importante analizar qué políticas o estrategias sientan las bases para el posterior diseño de la estrategia. En este estudio se arriba a la conclusión que el destino regional para trazar sus líneas estratégicas debe guiarse por la política contemplada en los Lineamientos de la política económica y social del Partido y la Revolución para el período 2016-2021 (PCC, 2017).

Del análisis del estado de la gestión del destino se puede concluir que actualmente el destino regional Villa Clara no cuenta con una estrategia formalizada para su desarrollo y gestión eficiente, donde para el futuro diseño de la estrategia se deberá tener en cuenta fuertes acciones de comercialización para lograr posicionar al destino en la mente del consumidor, en este caso de los turistas que lo visitan, además que esta estrategia deberá tener un enfoque integrador y sistémico para lograr la integración de todos los entes que intervienen en el proceso trabajando todos por la satisfacción de los implicados (tanto visitantes como población), así como ser consecuentes con los Lineamientos adoptados en la política económica y social y en el Plan nacional de desarrollo.

\section{Paso 8: Determinación de la fase actual del ciclo de vida del destino}

El modelo de Butler (1980), aún con las modificaciones posteriores señaladas, esencialmente basa su aplicación en la evolución de las llegadas de visitantes al destino (Coelho \& Lourenço, 2015). En la Figura 5 se refleja el número de llegadas de visitantes al destino desde el año 1992 con una marcada tendencia exponencial de crecimiento que han tenido los arribos durante estos años.

Mediante el análisis de ambas figuras (Figura 5 y Figura 6) se puede apreciar que, por la forma de las curvas, el destino turístico Villa Clara pudiera clasificarse en la fase de desarrollo, a pesar de que del año 2017 al 2018 haya ocurrido un descenso del número de visitantes al destino, por lo que la estrategia estaría encaminada al crecimiento diversificado. 


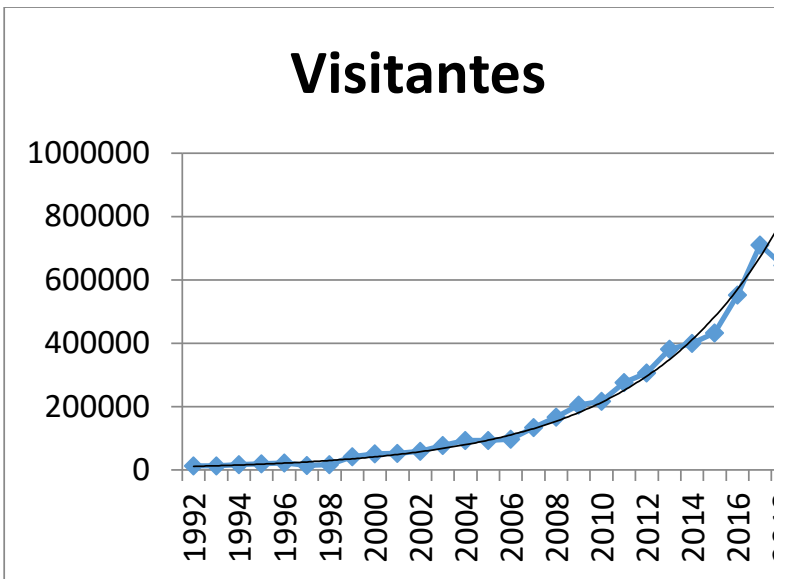

Fuente: Elaboración propia a partir de datos históricos del Mintur.

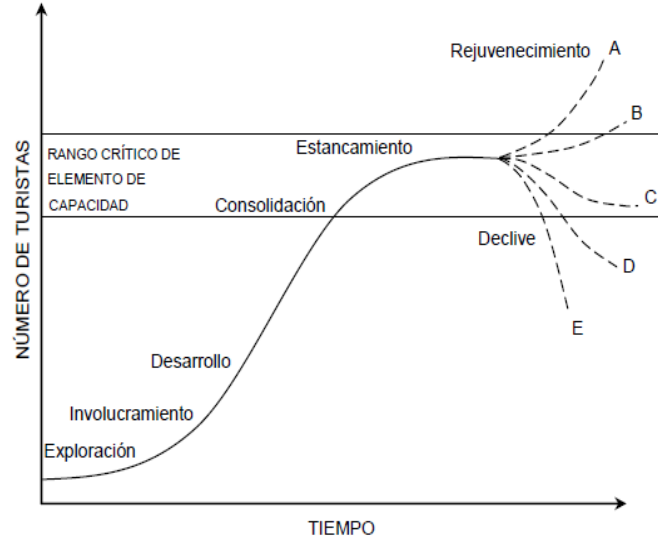

Fuente: Butler (1980).

\section{Etapa 6: Definición de los indicadores de gestión}

Rodríguez (2019) logra identificar cinco variables de importancia para el correcto funcionamiento del destino: ambiental, turística, de percepción, antrópica y económica. La variable ambiental tiene que ver con el aprovechamiento turístico de los aspectos medio ambientales propios del área; la turística con el desarrollo y uso turístico en el aspecto de equipamiento y servicios; la variable de percepción valora el grado de confort psicológico que un turista puede percibir en el área y la antrópica evalúa el potencial uso y desarrollo turístico de todas las manifestaciones humanas en la comarca, mientras que la económica vela por la generación de ingresos y el aumento de las inversiones. A cada una de estas variables se subscriben indicadores que evaluarán posteriormente las acciones a realizar como parte de la estrategia, es por ello que, se establecieron una serie de indicadores acordes a cada una de estas (Figura 7).

Figura 7. Indicadores de gestión por variables estratégicas

\begin{tabular}{|c|c|c|}
\hline Variables & Ind & ores \\
\hline \multirow{6}{*}{ 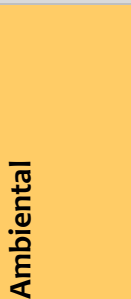 } & A1 & Certificaciones de calidad, ambientales \\
\hline & $\mathrm{A} 2$ & Trastornos ecológicos \\
\hline & A3 & Desastres naturales \\
\hline & A4 & Preservación del sistema natural/rural y litoral \\
\hline & A5 & Calidad de las playas \\
\hline & A6 & Capacidad de carga \\
\hline \multirow{5}{*}{ 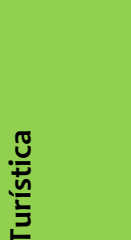 } & T1 & Número de llegadas turísticas \\
\hline & T2 & Porciento de crecimiento en las llegadas de turistas \\
\hline & T3 & Estancia media \\
\hline & T4 & Concentración por nacionalidades \\
\hline & T5 & Motivación del viaje \\
\hline
\end{tabular}




\begin{tabular}{|c|c|c|}
\hline & T6 & Estacionalidad de los flujos de turistas \\
\hline & T7 & Repetición o intención de retorno \\
\hline & T8 & Número de plazas y establecimientos disponibles \\
\hline & T9 & Evolución de la calidad de los medios de hospedaje (categorías) \\
\hline & T10 & Diversificación \\
\hline & T11 & Ubicación relativa (competidores, mercados emisores) \\
\hline & T12 & Grado de dependencia con los intermediarios \\
\hline & P1 & Imagen del destino \\
\hline & $\mathbf{P 2}$ & Congestión y trafico \\
\hline 으 & $\mathbf{P}_{3}$ & Percepción de los residentes sobre los turistas \\
\hline$\frac{0}{2}$ & P4 & Seguridad \\
\hline ư & $\mathrm{P}_{5}$ & Grado de satisfacción de los turistas \\
\hline 号 & P6 & Grado de interés de los atractivos \\
\hline & AN1 & Aumento de la urbanización \\
\hline & AN2 & Crecimiento de la población local \\
\hline & AN3 & Calidad de vida \\
\hline & AN4 & Necesidad de invertir en RRHH \\
\hline [3 & AN5 & Empleos generados \\
\hline$\frac{0}{0}$ & AN6 & Presión sobre el patrimonio cultural \\
\hline 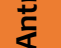 & AN7 & Población permanente/ número de turistas (Capacidad de carga social) \\
\hline & E1 & Aumento de las inversiones \\
\hline & E2 & Ingresos turísticos totales \\
\hline & E3 & Evolución de los precios ofertados \\
\hline. & E4 & Contribución del turismo al PIB \\
\hline 。ำ & E5 & Relación de otras actividades económicas con el turismo \\
\hline ợ & E6 & Rentabilidad del sector privado \\
\hline
\end{tabular}

Fuente: Rodríguez, 2019.

Asimismo, fueron determinados indicadores estratégicos capaces de evaluar varias variables (Figura 8). 
Figura 8. Indicadores Estratégicos

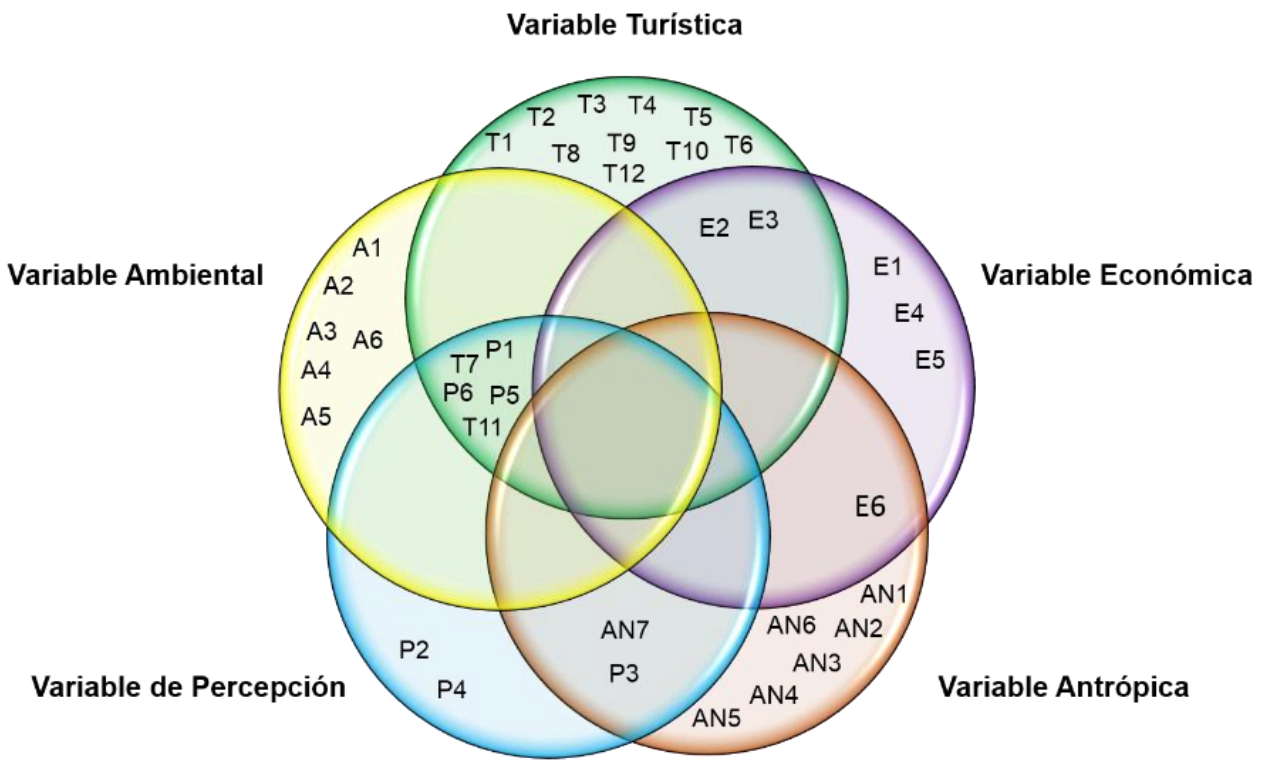

Fuente: Rodríguez, 2019.

\section{Etapa 7: Análisis estratégico}

Para diagnosticar la situación actual del destino, la autora utiliza una herramienta que sienta las bases para la planificación estratégica: la matriz de Debilidades, Amenazas, Fortalezas y Oportunidades (DAFO) a partir de todos los aspectos investigados. Se aplica la matriz según el método de impactos cruzados. El análisis DAFO evidencia que el destino se encuentra en el cuadrante MAXI-MAXI, por lo que se debe orientar la estrategia a aprovechar sus fortalezas y las oportunidades que le brinda el entorno (Rodríguez, 2019)

\section{- Fase III: Diseño de la estrategia}

\section{Etapa 8: Descripción de la estrategia}

La estrategia consta de una planificación de la gestión en el destino turístico regional Villa Clara, de forma integral y con enfoque sistémico, a partir de acciones trazadas por cada una de las Áreas de Resultados Claves (ARC) planteadas por Rodríguez (2016): investigación y desarrollo, comercialización, finanzas, logística y servucción. Esta estrategia se sustenta en el análisis DAFO realizado en la etapa anterior para el destino.

\section{Etapa 9: Planteamiento de los objetivos de la estrategia}

Propiciar la competitividad y diversificación en el destino regional Villa Clara, a partir de la vinculación de todos los actores que influyen, directa e indirectamente, en su desarrollo 
turístico; que involucre acciones de carácter sistémico, participativo, sostenible e integral dirigidas a la ampliación de la oferta con el impulso de modalidades con potencial en el territorio (turismo de naturaleza, histórico-cultural, de salud y de eventos e incentivo) y a la incorporación de nuevos mercados $\mathrm{y}$, dentro de ellos, también nuevos segmentos de mercado que impacten positivamente en la comunidad local y la forma de hacer turismo.

\section{Etapa 10: Orientación de la estrategia}

La estrategia está orientada a la diversificación, a partir de la gestión integral, con carácter sistémico, del destino turístico regional Villa Clara; considerando una línea base desde el año 2019 hasta el 2022 y una adaptación a las condiciones a partir del año 2023 a partir de escenarios prospectivos, con un horizonte temporal al año 2030.

\section{Etapa 11: Confección de la estrategia}

Para la realización de esta etapa se delimitan la misión y visión del destino turístico regional Villa Clara, las cuales van a servir de guía para el diseño del plan de acciones estratégicas.

\section{Etapa 12: Determinación del plan estratégico}

A partir de los resultados obtenidos se expresa como objetivo general: lograr la gestión estratégica integrada con enfoque sistémico en el destino turístico regional Villa Clara, basada en las fortalezas para el aprovechamiento de las oportunidades que le brinda el entorno. Para dar cumplimiento a este objetivo se establecen una serie de objetivos específicos por las Áreas de Resultados Claves (ARC) identificadas por Rodríguez (2016). En esta etapa se presentan los objetivos específicos por Área de Resultado Clave, las acciones para lograrlos, los recursos necesarios, los responsables encargados del desarrollo de las acciones y los plazos de ejecución acordes al tiempo definido en la línea de base, así como los indicadores de gestión que controlaran su correcta ejecución.

La realización de las fases IV y $\vee$ del procedimiento están en proceso de ejecución para el logro de la implementación de la estrategia que requiere de un período estimado de dos años, además, dentro del cronograma se establece que el periodo de tiempo planificado es de junio del 2019 a junio del 2022, y el cumplimiento de las mismas es potestad de la delegación del Mintur que ostenta la dirección del destino, la investigadora queda en función del acompañamiento, asesoría durante el proceso de ejecución, control y mejora.

\subsection{Valoración de los resultados de la aplicación del procedimiento}

La aplicación del procedimiento demostró la necesidad, importancia y actualidad del mismo para el desarrollo del destino turístico regional Villa Clara en particular, el cual puede ser generalizado para los destinos turísticos regionales cubanos. A través de la primera fase del mismo se logra la organización del trabajo, determinando las personas involucradas en su realización, el cronograma y el cumplimiento de las premisas de aplicación, constituyendo los cimientos para la continuidad del procedimiento. Del diagnóstico estratégico realizado se 
concluye que el diseño de una estrategia de gestión para el destino turístico regional Villa Clara es de vital importancia, repercutiendo en "el desarrollo local con el correspondiente impacto social por la generación directa e indirecta de empleos y la valoración de los atractivos turísticos del territorio" avalado por la delegación territorial del Mintur.

Con el diseño de estrategia de gestión para el destino turístico regional Villa Clara se logra establecer una estrategia adecuada a las características del destino, su misión y visión con los objetivos estratégicos y el plan estratégico diseñados, evidenciándose un resultado coherente y fácil de implementar, controlar y mejorar; lográndose con ello la necesaria retroalimentación. Desde el punto de vista económico se logra un impacto valorado por el ahorro de recursos materiales y financieros que se derivan de la implementación de estrategias adecuadas en el proceso de ejecución de los objetivos, la previsión del alcance hasta el 2022 se estima por la delegación provincial del Mintur en no menos de \$25 mil CUC.

\section{Conclusiones}

En el estudio de la gestión de los destinos turísticos se demostró que este proceso se apoya en la planificación estratégica como base para la toma de decisiones y proyección de estrategias que se centren en la satisfacción de los actores con base en los principios de la integración y del desarrollo sostenible. El procedimiento seleccionado para este fin posee cinco fases y 17 etapas, las cuales van desde la planificación y diseño del trabajo, incluyen la realización de un diagnóstico estratégico con ocho pasos para su ejecución, el diseño de la estrategia, así como la implementación de la misma con su correspondiente evaluación y mejora a través de los indicadores y variables estratégicas seleccionadas, posibilitando lograr un resultado científicamente aceptable.

La novedad científica de la investigación radica en concebir un procedimiento para el diseño de la estrategia de gestión de destinos turísticos regionales adaptado a las condiciones actuales del sector turístico cubano, así como a las condiciones de los destinos turísticos regionales en Cuba, en particular el destino Villa Clara, objeto de estudio; que permitirá a la alta dirección establecer acciones estratégicas basadas en estudios científicamente fundamentados y su posterior control y mejora.

La estrategia de gestión diseñada para el destino turístico regional Villa Clara logra que se gestione adecuadamente el destino turístico regional con un carácter de integración de los actores que intervienen aprovechando eficientemente sus recursos y atrayendo al mayor número de turistas al mismo de modo que satisfagan sus necesidades.

\section{Referencias}

Butler, R. W. (1980). The concept of tourism area cycle of evolution: Implications for management of resources. In R. Butler (Ed.), The Tourism Life Cycle, Vol. 1. Applications and modifications (pp. 3-12). Toronto: Channel View Publications.

Coelho, J. A. \& Lourenço, P. F. (2015). A identificação da fase do ciclo de vida de um destino turístico para as escolhas estratégicas: uma proposta de uma ferramenta expedita. Revista de Estudios Económicos y Empresariales, 27, 15-41. 
Colectivo de Autores. (2011). Gestión de destinos turísticos. Centro de Estudios Turísticos. Universidad de La Habana. La Habana.

De Arquer, M. I. (2006). Fiabilidad humana. Métodos. Centro nacional de condiciones. Retrieved from https://www.insst.es/documents/94886/326827/ntp_377.pdf/dc0902f5-d3e8-4b3f-817cob971416ee1d

López, J.M. \& López, L.M. (2007). Diferencias territoriales en la planificación y la gestión del destino turístico. En Cuadernos de Turismo, 19, 71-90. Universidad de Murcia.

Machado, E., (2011) Diseño de productos turísticos integrados. Aplicación en la región central del destino Cuba (doctoral thesis). Universidad Central Marta Abreu de las Villas, Cuba.

Martín, R. (2010). Principios, organización y práctica del turismo. La Habana: Editorial Félix Varela.

Navarro, Y. (2012). Proyección del turismo de eventos en el destino turístico Villa Clara (trabajo de diploma). Universidad Central "Marta Abreu" de las Villas. Santa Clara, Cuba.

OMT [Organización Mundial del Turismo]. (1998). Introducción al turismo. Madrid: OMT.

Partido Comunista de Cuba. (2017). Documentos del $7 \mathrm{mo}$. Congreso del Partido aprobados por el III Pleno del Comité Central del PCC el 18 de mayo de 2017 y respaldados por la Asamblea Nacional del Poder Popular el 1 de junio de 2017. En Periódico Granma, Tabloide I. La Habana, Cuba.

Porter, M.E. (1985). Estrategia Competitiva: Técnicas para el análisis de los sectores industriales y de la competencia. México: Editorial Continental.

Rodríguez, G. (2016). Determinación de la fase del ciclo de vida del destino turístico regional Villa Clara y de los factores influyentes en su estrategia de crecimiento (trabajo de diploma). Universidad Central "Marta Abreu" de las Villas, Santa Clara, Cuba.

Rodríguez, G. (2019). Procedimiento para el diseño de la estrategia de gestión de destinos turísticos regionales: Caso destino Villa Clara (tesis de maestría). Universidad Central "Marta Abreu" de las Villas, Santa Clara, cuba.

Rodríguez, R. (2005) Gestión de Destinos Turísticos. Texto docente Centro de Estudios Turísticos. Editado por EAEHT, Universidad de la Habana.

Rodríguez, R. (2011). Destinos turísticos. Realidad y concepto. TURyDES, 4(11).

Salinas, E. (2003). Geografía y Turismo. Aspectos territoriales del manejo y gestión del turismo. La Habana: Ediciones SI-MAR.

Valls, J., (2004) Gestión de destinos turísticos sostenibles. Madrid: Gestión 2000.

GISELLE RODRÍGUEZ JIMÉNEZ: Licenciada en Turismo. Máster en Gestión Turística. Profesora e investigadora del Departamento de Turismo de la Universidad Central "Marta Abreu" de Las Villas, Cuba. Especialista en Gestión de Destinos Turísticos. Miembro de la Sociedad Nacional de Marketing en Cuba y de la Red Caribeña de Turismo Responsable. 2. Dirección postal: Universidad Central "Marta Abreu" de Las Villas, Facultad de Ciencias Económicas, Departamento de Turismo, Carretera de Camajuaní Km 5 1⁄2 CP 54830, Santa Clara, Villa Clara, Cuba.

ALICIA DE LA CONCEPCIÓN ALFONSO SERAFíN: Licenciada en psicología. Doctora en Ciencias Pedagógicas. Profesora e investigadora universitaria desde 1974, actualmente se desempeña como profesora consultante del Departamento de Turismo de la Universidad Central "Marta Abreu" de las Villas, Cuba. Miembro de la Asociación Nacional de Economistas y Contadores de Cuba. 2. Dirección 
postal: Universidad Central "Marta Abreu" de Las Villas, Facultad de Ciencias Económicas, Departamento de Turismo, Carretera de Camajuaní Km 5 1⁄2 CP 54830, Santa Clara, Villa Clara, Cuba.

CARLOS CRISTÓBAL MARTÍNEZ MARTÍNEZ: Ingeniero Industrial. Doctor en Ciencias Económicas. Especialista en Dirección. Profesor e investigador universitario desde 1979. Más de 75 publicaciones en revistas indexadas. Profesor de Dirección en universidades de: Cuba, México, Colombia, Venezuela, Chile y Ecuador. Jefe del Departamento Turismo de la Universidad Central "Marta Abreu” de Las Villas, Cuba. 2. Dirección postal: Universidad Central "Marta Abreu" de Las Villas, Facultad de Ciencias Económicas, Departamento de Turismo, Carretera de Camajuaní Km 5 1⁄2 CP 54830, Santa Clara, Villa Clara, Cuba.

Submitted on January 22, 2020

Accepted on April 7, 2020 